\title{
Generation of a BAC transgenic mouse strain that expresses CreERT and a fluorescent protein under the transcriptional control of the Fzd5 locus
}

Satoru Miyagi ${ }^{1{ }^{*}}$, Yuko Kato ${ }^{1,2+}$, Ayako Watanabe ${ }^{1}$, Kenichi Miyamoto ${ }^{1}$, Rintaro Yoshikawa ${ }^{1}$, Keita Hagiya ${ }^{3}$, Daisuke Hirano ${ }^{3}$ and Yumi Matsuzaki ${ }^{1,2^{*}}$

\begin{abstract}
Background: The expression of FZD5 distinguishes immature human mesenchymal stem/stromal cells (MSC) in cultures, and the function of FZD5 is crucial for maintaining the proliferation and multilineage differentiation capacity of human MSC. We herein investigated whether Fzd5 expression also marks undifferentiated MSC in animals.
\end{abstract}

Methods: We generated a transgenic mouse strain (Fzd5-CreERT-tFP635) that expresses CreERT and the fluorescent protein, TurboFP635 (tFP635), under the transcriptional control of the Fzd5 gene using the BAC transgenic technique, and identified cells expressing tFP635 by flow cytometry. We also conducted lineage tracing with this strain.

Results: In the bone marrow of transgenic mice, tFP635 was preferentially expressed in MSC, Leptin receptorexpressing MSC (LepR ${ }^{+} \mathrm{MSCs}$ ), and some Pdgfra ${ }^{+} \mathrm{Sca}^{+}{ }^{+} \mathrm{MSC}$ (PaS). Inducible lineage tracing using the Fzd5-CreERTtFP635; CAG-CAT-EGFP strain at the adult stage showed that Fzd5-expressing cells and their descendants labeled with GFP were progressively dominant in LepR ${ }^{+} M S C$ and PaS, and GFP ${ }^{+}$cells persisted for 1 year after the activation of CreERT. Adipocyte progenitor cells (APCs), osteoblast progenitor cells (OPCs), and Cd51 ${ }^{+}$stromal cells were also labeled with GFP.

Conclusions: Our transgenic mouse marks two different types of MSC, LepR ${ }^{+} M S C$ and PaS.

Keywords: Mesenchymal stem/stromal cells (MSC), Fzd5, PaS, Leptin receptor-expressing MSC (LepR ${ }^{+}$MSC)

\section{Background}

Mesenchymal stem/stromal cells (MSCs) self-renew and differentiate into mesenchymal lineages and reside in the bone marrow (BM) and other tissues. MSC have been isolated by plastic adherence and subsequent cultivation since the 1970s $[1,2]$. In the last two decades, researchers have developed a strategy to prospectively

\footnotetext{
*Correspondence: miyagi@med.shimane-u.ac.jp; matsuzak@med.shimaneu.ac.jp

†Satoru Miyagi and Yuko Kato contributed equally to this work. 'Department of Life Science, Faculty of Medicine, Shimane University, 89-1 Enya, Izumo, Shimane 693-8501, Japan

Full list of author information is available at the end of the article
}

isolate MSC from mouse BM and other tissues using monoclonal antibodies and/or reporter mice and identified various MSC fractions in vivo [3]. BM reticular cells expressing high levels of CXCL12 have been identified as CXCL12-abundant reticular cells (CAR cells), which preserve their self-renewal capacity and multilineage differentiation capacity in vitro and exhibit a lifelong selfrenewal capacity in vivo. Histochemical analyses and cell ablation experiments revealed that CAR cells are an important source of niche factors that are required for the maintenance of hematopoietic stem cells (HSCs) [4, 5]. BM stromal cells, which express the leptin receptor

(c) The Author(s). 2022 Open Access This article is licensed under a Creative Commons Attribution 4.0 International License, which permits use, sharing, adaptation, distribution and reproduction in any medium or format, as long as you give appropriate credit to the original author(s) and the source, provide a link to the Creative Commons licence, and indicate if changes were made. The images or other third party material in this article are included in the article's Creative Commons licence, unless indicated otherwise in a credit line to the material. If material is not included in the article's Creative Commons licence and your intended use is not permitted by statutory regulation or exceeds the permitted use, you will need to obtain permission directly from the copyright holder. To view a copy of this licence, visit http://creativecommons.org/licenses/by/4.0/. 
(LepR $\left.{ }^{+} \mathrm{MSC}\right)$, contribute to adipogenesis and osteogenesis in BM [6, 7]. LepR ${ }^{+}$MSC is also a component of the perisinusoidal niche and express the niche factors, Kitl and Cxcl12. Functionally, the conditional deletion of Kitl or Cxcl12 in $\mathrm{LepR}^{+} \mathrm{MSC}$ leads to the depletion or mobilization of $\mathrm{HSC}$, respectively, in BM. Therefore, LepR $^{+}$MSC is critical for the maintenance of HSC and retention of HSC in the BM. A recent comparative analysis revealed that CAR cells and LepR ${ }^{+}$MSCs were substantially overlapping populations [8-10]. Cd $51^{+} \mathrm{Pdgfr \alpha}^{+}$ BM stromal cells form mesenspheres with a capacity for mesenchymal lineage differentiation. $\mathrm{NG}^{+}$Nestin$G F P^{\text {bright }}$ periarteriolar cells also maintain MSC properties, and $\mathrm{Cd} 51^{+} \mathrm{Pdgfr \alpha}{ }^{+}$stromal cells are a subpopulation of Nestin-GFP $P^{+}$cells [11]. On the other hand, cultured MSCs exert immunomodulatory functions through various pathways and have been applied to clinical treatments $[12,13]$. Accordingly, beyond the homeostasis of the mesenchymal cell population, BM-MSC are involved in the regulation of hematopoiesis and immune responses through the secretion of various cytokines and chemokines.

To date, various MSC fractions have been identified in mouse BM, and the function(s) of each BM-MSC are being revealed. However, many questions remain unanswered in MSC biology, such as whether known MSCs originate from a common "bona fide undifferentiated MSC," if a hierarchical relationship exists between known MSCs, and how the proliferation, differentiation, and function(s) of each MSC are controlled. We previously demonstrated that mouse BM stromal cells expressing Pdgfr $\alpha$ and Scal, named P $\alpha S$, are highly enriched MSC with a high proliferative capacity and differentiation potential toward a mesenchymal lineage in vitro and in the transplantation setting [14]. We recently performed extensive gene expression profiling on human MSC clones and showed that Frizzled5 (FZD5), a receptor for the WNT signaling molecule, was exclusively expressed in highly functional and immature human MSC clones. Gain- and loss-of-function analyses revealed that FZD5 negatively regulated cellular senescence in human MSC, thereby maintaining proliferation and multilineage potency [15].

To investigate whether the expression of Fzd5 marks immature MSC in mouse, we herein generated a reporter mouse strain (Fzd5-CreERT-tFP635) that expresses CreERT and fluorescent protein, TurboFP635 (tFP635), under the control of the transcriptional regulatory sequence of the $F z d 5$ gene using BAC transgenic technology. In the BM of transgenic mice, tFP635 is preferentially expressed in $\mathrm{LepR}^{+} \mathrm{MSC}$ and a part of $\mathrm{P} \alpha \mathrm{S}$. A lineage tracing experiment with Fzd5-CreERT-tFP635; CAG-CAT-EGFP showed that LepR ${ }^{+} \mathrm{MSC}$ and P $\alpha \mathrm{S}$ were labeled with GFP, the frequency of $\mathrm{GFP}^{+}$cells in these fractions increased over time after the activation of CreERT, and $\mathrm{GFP}^{+}$cells were maintained for up to 1 year, showing that our transgenic mouse marks two types of MSC, LepR ${ }^{+}$MSC, and P $\alpha$.

\section{Methods}

Generation of BAC transgenic mice

Fzd5-CreERT-tFP635 were generated using the BAC transgenic technique. The coding sequence of the $F z d 5$ gene in the BAC clone, RP23-142C12, was replaced with a 4.3 Kb DNA fragment containing CreERT and three copies of tFP635 cDNA in Escherichia coli using the Red/ET Recombination System (Gene Bridges). Recombined BAC DNA was then injected into pronuclei and transplanted into pseudopregnant females. Ten independent founder lines were obtained after screening 60 F0 mice with Southern blotting using the Cre cDNA probe, and the lines A, F, G, H, and I were described herein.

\section{Mice}

B6.129(Cg)-Lepr ${ }^{\text {tm2(cre) }}$ Rck/J (LepR-Cre, JAX lab \#008320) and B6.Cg-Gt(ROSA)26Sor ${ }^{\text {tm 14(CAG-tdTomato)Hze, }}$ J (ROSA-CAG-LSL-tdTomato, JAX lab \#007914) were purchased from the Jackson Laboratory and backcrossed at least six times onto a C57BL/6J background. CAGCAT-EGFP mice [16] were kindly provided by Dr. Junichi Miyazaki (Osaka University, Osaka, Japan). All mice were maintained in the C57BL6J background.

In lineage tracing experiments, Fzd5-CreERT-tFP635 or LepR-Cre were crossed with CAG-CAT-EGFP or ROSA-CAG-LSL-tdTomato, respectively, and compound mice were obtained. To induce CreERT activity in Fzd5CreERT-tFP635; CAG-CAT-EGFP, 8- to 12-week-old mice were intraperitoneally injected with $100 \mu \mathrm{l}$ of tamoxifen (TM) dissolved in corn oil at a concentration of $10 \mathrm{mg} / \mathrm{ml}$ for 3 or 5 consecutive days.

\section{Preparation of femur sections}

The femur was cryosectioned using the Kawamoto film method [17].

\section{Preparation of BM cells for FCM and cell sorting}

$\mathrm{P} \alpha \mathrm{S}$ is enriched in collagenase-released cells (CR) - the cells released from bone fragments with collagenase digestion [14]. In contrast, LepR ${ }^{+}$MSC comprise some reticular cells and reside in BM. Therefore, a femur and tibia pair was processed to prepare a single-cell suspension containing $\mathrm{CR}$ for the detection of $\mathrm{P \alpha S}$, and the other pair was used to prepare BM and detect LepR ${ }^{+}$MSC. To prepare the single-cell suspension for $\mathrm{P} \alpha \mathrm{S}$ staining, the femur and tibia were minced with scissors and incubated at $37^{\circ} \mathrm{C}$ for $30 \mathrm{~min}$ in collagenase solution [DMEM (Wako Chemicals) containing 0.2\% collagenase (Wako Chemicals), 25 units $/ \mathrm{mL}$ 
deoxyribonuclease I (Sigma-Aldrich), 10 mM HEPES, and $1 \%$ penicillin/streptomycin]. Bone fragments were tapped with a mortar and pestle 50-100 times and filtered with a cell strainer (Falcon 2350) to remove debris and bone pieces. Cells were collected by centrifugation at $300 \times g$ at $4^{\circ} \mathrm{C}$ for $5 \mathrm{~min}$, hemolyzed, and washed with FACS buffer (PBS supplemented with 2\% FBS). Regarding LepR ${ }^{+}$MSC staining, BM cells were isolated by flushing from femurs and tibias, followed by enzyme digestion with collagenase and deoxyribonuclease I solution at $37^{\circ} \mathrm{C}$ for $20 \mathrm{~min}$. After hemolysis, BM cells were washed with FACS buffer and filtered.

\section{FCM and cell sorting}

FCM and cell sorting were performed using antibodies recognizing the following antigens: Cd45 (30-F11), Ter119 (TER-119), Pdgfra (APA5), Sca1 (D7), Cd31(MEC13.3), Cd51 (HM $\alpha \mathrm{V}-1)$, Cd24 (M1/69 or 30F1), and the leptin receptor (goat polyclonal antibody). These antibodies were purchased from eBioScience, BioLegend, TONBO, and R\&D Systems. Dead cells were eliminated by staining with $1 \mu \mathrm{g} / \mathrm{mL}$ propidium iodide

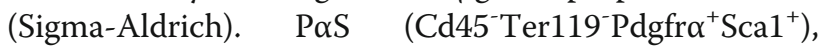
LepR ${ }^{+}$MSC $\quad\left(\right.$ Cd $_{4} 5^{-}$Ter119-Cd31-Sca1 ${ }^{-}$LepR $\left.^{+}\right), \quad$ APC $\left(\mathrm{Cd} 45^{-} \mathrm{Ter} 119^{-} \mathrm{Cd} 31^{-} \mathrm{Cd} 24^{-} \mathrm{Scal}^{+}\right), \quad$ OPC $\left(\mathrm{Cd} 45^{-}\right.$Ter119 $\left.{ }^{-} \mathrm{Cd} 31^{-} \mathrm{Pdgfra}^{+} \mathrm{Sca} 1^{-}\right)$, and $\mathrm{Cd} 51^{+}$stromal cells $\left(\mathrm{Cd} 45^{-} \mathrm{Ter} 119^{-} \mathrm{Cd} 31^{-} \mathrm{Cd} 51^{+}\right)$were defined as described elsewhere. The combination of antibodies was as follows:

PaS+tFP635; Biotin-anti-Pdgfro, Fluorescein isothiocyanate (FITC)-anti-Sca1, Phycoerythrin (PE)-anti-Cd45, PE-anti-Ter119, and Allophycocyanin (APC)-Streptavidin. PoS+GFP; PE-anti-Pdgfro, APC-anti-Cd45, APCanti-Ter119, and Brilliant violet 421 (BV421)-anti-Sca1. LepR $^{+}$MSC+tFP635; Biotin-anti-Pdgfro, FITC-antiSca1, PE-anti-Cd45, PE-anti-Ter119, PE-anti-Cd31, and APC-Streptavidin. LepR ${ }^{+}$MSC+GFP; Biotin-anti-LepR, PE-anti-Cd31, APC-Cy7-anti-Cd45, APC-Cy7-antiTer119, PE-Cy7-anti-Sca1, and BV421-Streptavidin. APC/OPC+GFP; Biotin-anti-Cd24, PE-Streptavidin, APC-anti-Pdgfro, APC-Cy7-anti-Cd45, APC-Cy7-antiTer119, PE-Cy7-anti-Cd31, and BV421-anti-Sca1. Cd51 ${ }^{+}$Stroma+GFP; Biotin-anti-Cd51, PE-Streptavidin, APC-Cy7-anti-Cd45, APC-Cy7-anti-Ter119, and BV421anti-Cd31. Sorting of PoS subfractions; Biotin-antiPdgfra, FITC-anti-Sca1, PE-anti-Cd45, PE-anti-Ter119, and APC-Streptavidin.

Cell sorting and the analysis of tFP635 expression were performed on MoFlo (Beckman Coulter Life Sciences) equipped with a yellow-green laser, while other analyses were conducted on CytoFLEX (Beckman Coulter Life Sciences).

\section{MSC adherent culture}

Sorted cells were cultured in growth medium (DMEM [Wako chemicals] supplemented with 20\% FBS, $10 \mathrm{mM}$ HEPES, and antibiotics) and incubated at $37^{\circ} \mathrm{C}$ with $5 \%$ $\mathrm{CO}_{2}$. Medium was replenished every $3-4$ days.

\section{CFU-F assay}

Approximately 500 sorted cells were seeded on a 100$\mathrm{mm}$ dish in growth medium. A colony was defined as a cluster of 50 or more cells.

\section{Differentiation cultures}

Adipogenic differentiation was induced using hMSC Adipogenic differentiation Medium Bullet Kit (LONZA) for 6 days, while osteogenic differentiation was induced with osteogenic media (DMEM supplemented $10 \mathrm{mM} \beta$ glycerophosphate, $50 \mu \mathrm{M} \mathrm{L}$-ascorbic acid, and $1 \mathrm{mM}$ dexamethasone) for 21 days. Cells were fixed with $4 \%$ paraformaldehyde for $10 \mathrm{~min}$ and stained with either Oil Red $\mathrm{O}$ or Alizarin Red according to standard protocol, and then images were acquired with a BZ-X700 fluorescent microscope (Keyence). After conversion to black and white image, the area with stains was quantified with ImageJ software.

\section{Quantitative RT-PCR}

The total RNA was extracted using the RNeasy Micro Kit (Qiagen, Hilden, Germany) and reverse-transcribed by the PrimeScript Reverse Transcriptase (TaKaRa Bio, Japan) with a random hexamer primer. Real-time quantitative PCR was performed with the SYBR Green PCR Master Mix (ThermoFisher Scientific). The primer sequences used were as follows:

Hprt1, 5'-TCCTCCTCAGACCGCTTTT-3', and 5'CCTGGTTCATCATCGCTAATC-3'; $F z d 5$, 5'-CAGCAGGATCCTCCGAGA-3', and 5'-CAGCACTCAGTT CCACACCA-3'; Cxcl12, 5'-CCAGAGCCAACGTCAA GCAT-3', and 5'-CAGCCGTGCAACAATCTGAA-3'; Kitl, 5'-GGTAGCTAGTTCTATCCATGCGGT-3', and 5'-CCTGTAAGGACTTTTCTGGAGAGTCT-3'; FoxC1, 5'-CACTCGGTGCGGGAAATGT-3', and 5'-GGTA CAGAGACTGACTGGCA-3'; Lepr, 5'-GTTCCAAACC CCAAGAATTG-3', and 5'-TGATTCTGCATGCTTG GTAAA-3'; Nes, 5'-TCCCTTAGTCTGGAAGTGGC TA-3', and 5'-GGTGTCTGCAAGCGAGAGTT-3'; Cspg4, 5'-GCAGAGGAGGTCTTGGTGAA-3', and 5'GGACATCTCGTGCTCATACAGA-3'.

\section{Statistical analysis}

Statistical analyses were performed using Graph Pad Prism version 9. The significance of differences was measured by the Student's $t$ test. Data are shown as the mean \pm SEM. Significance was taken at values of * $p$ less 
than $.05, * * p$ less than $.01,{ }^{* * * *} p$ less than $.005,{ }^{* * * * *} p$ less than .0005 , and ${ }^{* * * * * *} p$ less than .00005 .

\section{Results}

\section{Generation of BAC transgenic mice}

We previously reported that FZD5 was preferentially expressed in MSC clones with a high proliferative capacity and multilineage differentiation capacity in humans. FZD5-mediated WNT signaling functions as a critical negative regulator of cellular senescence. The inhibition of FZD5 with short hairpin RNA led to premature cellular senescence [15]. These findings raised the question of whether the expression of Fzd5 also marks immature MSC in vivo. Therefore, we generated transgenic mouse strains using a BAC clone, in which the coding sequence of $F z d 5$ was replaced with $C r e E R T$ and 3 copies of $t F P 635$ cDNA linked with P2A sequences (Fig. 1A). Five Fzd5-CreERT-tFP635 transgenic lines (Fzd5-CreERT-tFP635A, F, G, H, and $I$ line) from 10 independent founders were generated and backcrossed to C57BL6J mice more than 3 times to confirm transgene inheritance before assessments of BAC DNA copy numbers and an expression analysis of tFP635. Quantitative genomic PCR using primers, which amplify a 5 '-UTR region in both the endogenous and exogenous $\mathrm{Fzd} 5$ gene, revealed the copy numbers of BAC DNA (Fig. 1B).

\section{Localization of tFP $635^{+}$cells in the femur}

To examine the anatomical localization of $\mathrm{tFP} 635^{+}$cells in $\mathrm{BM}$, we performed a histological analysis of femur sections from Fzd5-CreERT-tFP635H and found that tFP635 ${ }^{+}$cells were abundant in the epiphysis and to a lesser extent in the diaphysis. tFP635 $5^{+}$cells showed two distinct localization patterns: cells lining the endosteum or those uniformly distributed to BM (Fig. 1C). We also observed the similar localization pattern of $\mathrm{tFP} 635^{+}$cells in the femur sections from Fzd5-CreERT-tFP635I (Additional file 1). These results demonstrated that at least two types of cells express tFP635 in Fzd5-CreERTtFP635.

\section{Optimization of the MSC preparation protocol}

Since the anatomical localization of BM-MSC in long bones, e.g., the tibia and femur, is unique to each MSC subtype, we optimized the protocol to prepare a singlecell suspension for a flow cytometric analysis (FCM) of two identified BM-MSCs, P $\alpha S$ (Cd45-Ter119-Pdg$\left.\mathrm{fro}^{+} \mathrm{Scal}^{+}\right)$, and $\mathrm{LepR}^{+} \mathrm{MSC} \quad\left(\mathrm{Cd} 45^{-} \mathrm{Ter} 19^{-} \mathrm{Cd} 31^{-} \mathrm{S}-\right.$ ca1 ${ }^{-} \operatorname{Pdgfr} \beta^{+}$Lepr $\left.^{+}\right)$. To achieve this, dissected long bones were initially divided into 2 parts, the bone ends $(\mathrm{BE})$, and bone shaft, then the shaft was flushed out to obtain "marrow". Residual bone shaft (bone), BE, and unfractionated whole bone (WB) were separately minced and digested with collagenase and subjected to FCM
(Additional file 2A, B and C). The frequency of $\mathrm{P} \alpha \mathrm{S}$ was the highest in $\mathrm{BE}$, and absolute number of $\mathrm{P} \alpha \mathrm{S}$ in $\mathrm{BE}$ was equivalent to that of $\mathrm{WB}$, suggesting that $\mathrm{P} \alpha \mathrm{S}$ mainly resided in $\mathrm{BE}$ (Additional file $2 \mathrm{~B}, \mathrm{D}$ and $\mathrm{E}$ ). Pdgfr $\alpha$ single-positive cells (PDSP) and Sca1 singlepositive cells (SSP) were distributed in all four fractions to some extent, and the absolute number was the most abundant in WB (Additional file $2 \mathrm{~B}, \mathrm{D}$ and $\mathrm{E}$ ). In contrast, LepR ${ }^{+}$MSC exclusively resided in marrow (Additional file 2C, D and E). Notably, we noticed the $\mathrm{Lepr}^{+}$ non-hematopoietic/endothelial

$\left(\mathrm{Cd} 45^{-} \mathrm{Ter} 119^{-} \mathrm{Cd} 31^{-} \mathrm{Sca} 1^{-}\right)$cells contained both Pdgfr $\beta^{+}$ and Pdgfr $\beta^{-}$cells. The latter was abundant in bone fraction or to some extent in $\mathrm{BE}$ and $\mathrm{WB}$, whereas in the marrow, the Lepr ${ }^{+}$cells were predominantly positive for Pdgfr $\beta$ (Additional file 2C). Based on these results, we prepared WB fractions from one leg to detect $\mathrm{P} \alpha \mathrm{S}$ and marrow from the other leg to identify LepR ${ }^{+}$MSCs that defined as Cd $45^{-} \mathrm{Ter} 119^{-} \mathrm{Cd} 31^{-} \mathrm{Sca} 1^{-} \mathrm{Lepr}^{+}$cells in subsequent experiments.

\section{tFP635 expression in Fzd5-CreERT-tFP635}

To test the expression pattern of tFP635, we conducted FCM of WB and marrow from Fzd5-CreERT-tFP635I mice. In the WB fraction, tFP635 ${ }^{+}$cells were mainly located in the PDSP region, followed by the double negative (DN) and $\mathrm{P} \alpha \mathrm{S}$, and were negligible in the SSP region (Fig. 2A). In the marrow, approximately $90 \%$ of tFP $635^{+}$ cells were in $\mathrm{Scal}^{-} \mathrm{Lepr}^{+}$region (Fig. 2B). All five strains tested in the present study showed similar results with some variations (Fig. 2C, D). We noted that tFP635 was also expressed in some $\mathrm{Cd} 45^{+}$or Ter $119^{+}$hematopoietic cells (HC) (data not shown). In reverse gating, approximately $10 \%$ of P $\alpha$ S, $40-90 \%$ of PDSP, less than $1 \%$ of SSP/DN, and $90 \%$ of LepR ${ }^{+}$MSC (except for line F) were positive for tFP635 (Fig. 2E). In summary, cells expressing tFP635 largely overlapped with LepR ${ }^{+}$MSCs but less with PDSP. In other words, PDSPs contain more tFP635-negative cells than LepR ${ }^{+}$MSCs. So, we focused on tFP635-negative cells in the PDSPs. In an additional analysis (Fig. 2F, see also Additional file 3 for full FCM profiles), approximately $80 \%$ of PDSP were positive for tFP635 and most of them were also positive for Lepr, while the cells negative for tFP635 expression were predominantly negative for Lepr. These results showed that PDSP contains three subsets that are $\mathrm{tFP} 65^{+} \mathrm{LepR}^{+} \mathrm{MSC}$, $\mathrm{tFP} 65^{+}$Lepr $^{-}$, and $\mathrm{tFP} 635^{-} \mathrm{Lepr}^{-}$. In $\mathrm{P \alpha S}$, more than $80 \%$ were negative for both tFP635 and Lepr (Fig. 2F-H). RT-qPCR revealed that tFP635 ${ }^{+}$PDSP expressed not only Fzd5 and Lepr, but also the LepR $^{+}$MSC markers, Cxcl12, Kitl, and FoxC1 $[18,19]$ (Fig. 2I). Collectively, these results showed that tFP635 ${ }^{+}$PDSP are LepR ${ }^{+}$MSC. In addition, RT-qPCR of WB fractions revealed that Fzd5 expression is highest in 


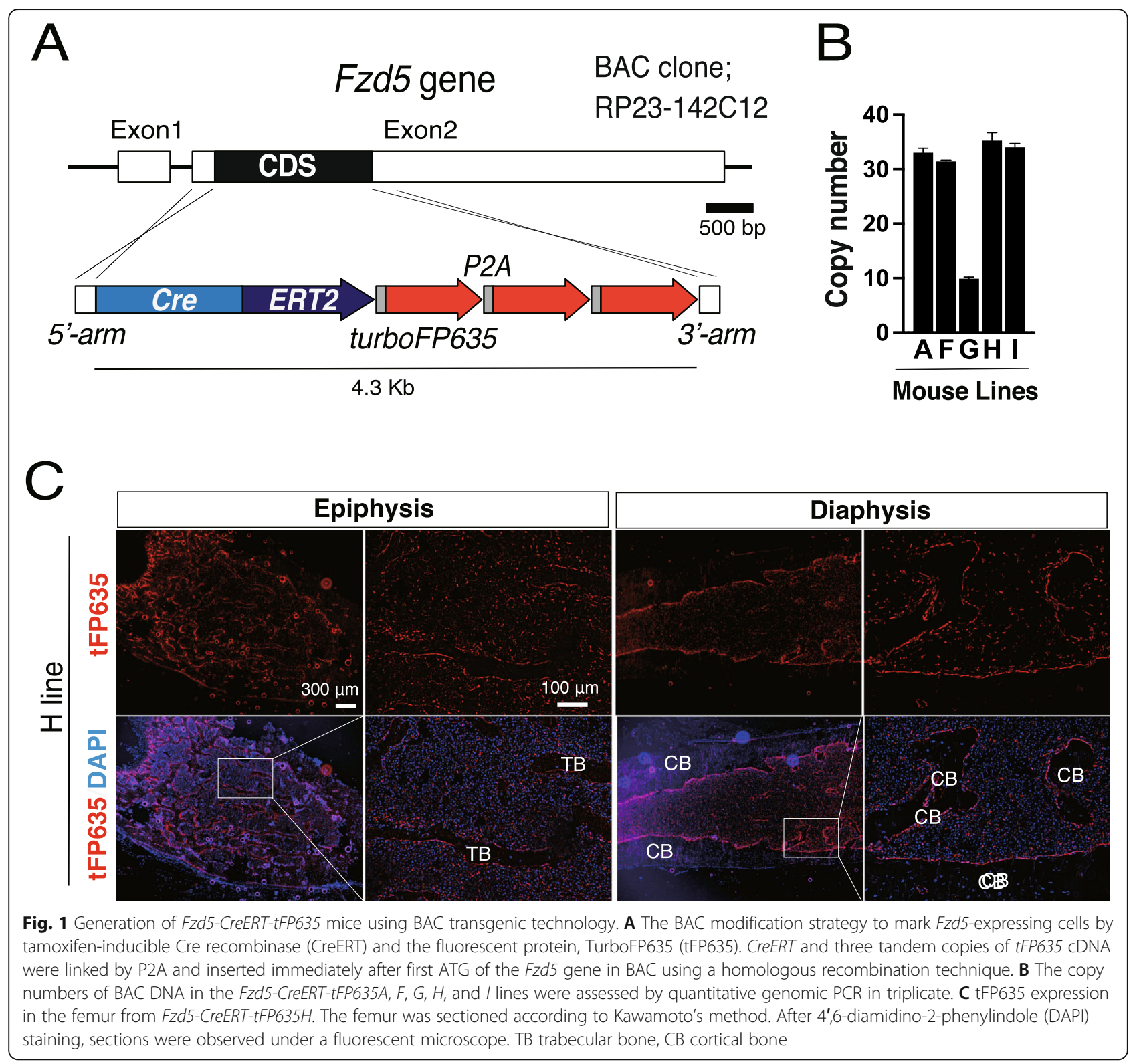

PDSP, and followed by $\mathrm{P} \alpha \mathrm{S}$, showing that the expression of tFP635 reflects endogenous Fzd5 expression (Additional file 4). Therefore, the expression of Fzd5 was mostly restricted to $\mathrm{LepR}^{+} \mathrm{MSC}$ and a part of $\mathrm{P} \alpha \mathrm{S}$ in the BM of Fzd5-CreERT-tFP635.

\section{Cell fate of Fzd5-expressing cells}

To trace the fate of $F z d 5$-expressing cells, mainly LepR ${ }^{+}$MSC and P $\alpha$, we crossed Fzd5-CreERT-tFP635I with CAG-CAT-EGFP, in which a loxP-flanked CAT-pA cassette impedes CAG promoter-driven GFP expression [16] and generated Fzd5-CreERT-tFP635; CAG-CATEGFP (hereafter referred to as Fzd5-GFP). In the Fzd5$G F P$, the activation of CreERT irreversibly excised the cassette and induced the expression of GFP in Fzd5- expressing cells and their descendants (Fig. 3A). Fzd5GFP were injected with TM for 3 or 5 consecutive days at between 8 and 12 weeks old to induce CreERT activity, and GFP expression was monitored in stromal fractions for 12 months (Fig. 3B-D). On day 4, approximately $10 \%$ of $\mathrm{P} \alpha \mathrm{S}$ were labeled with GFP, and GFP positivity and intensity progressively increased over time. The GFP positivity was maintained at approximately $80 \%$ for up to 12 months after the TM injection (Fig. 3B, D), whereas tFP635 along with CreERT was only expressed by a small portion of $\mathrm{P} \alpha \mathrm{S}$ cells (Fig. 2A, $\mathrm{C}, \mathrm{E})$. The proportion of $\mathrm{GFP}^{+}$cells in SSP also increased with similar kinetics to $\mathrm{P} \alpha \mathrm{S}$ despite the lack of obvious tFP635 expression in SSP (Fig. 2A, C, E and Fig. $3 \mathrm{D})$. In the marrow, approximately $30 \%$ of $\mathrm{LepR}^{+} \mathrm{MSC}$ 

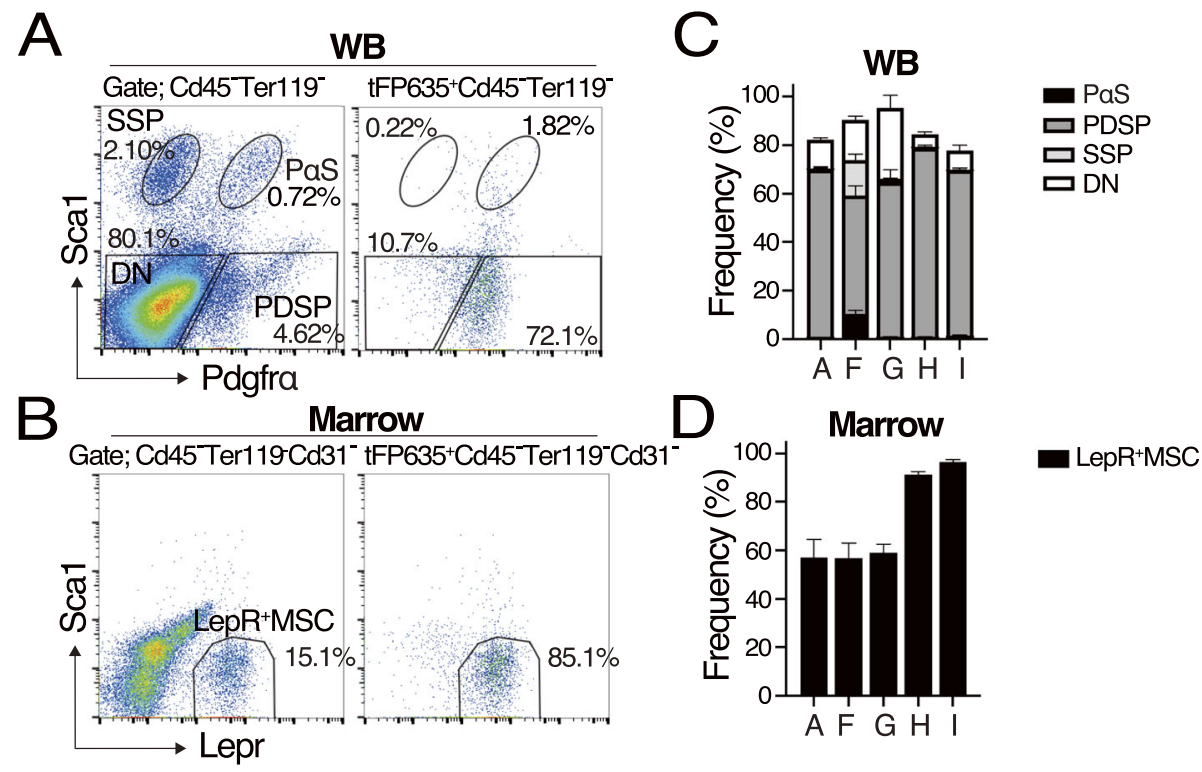

$\mathrm{E}$
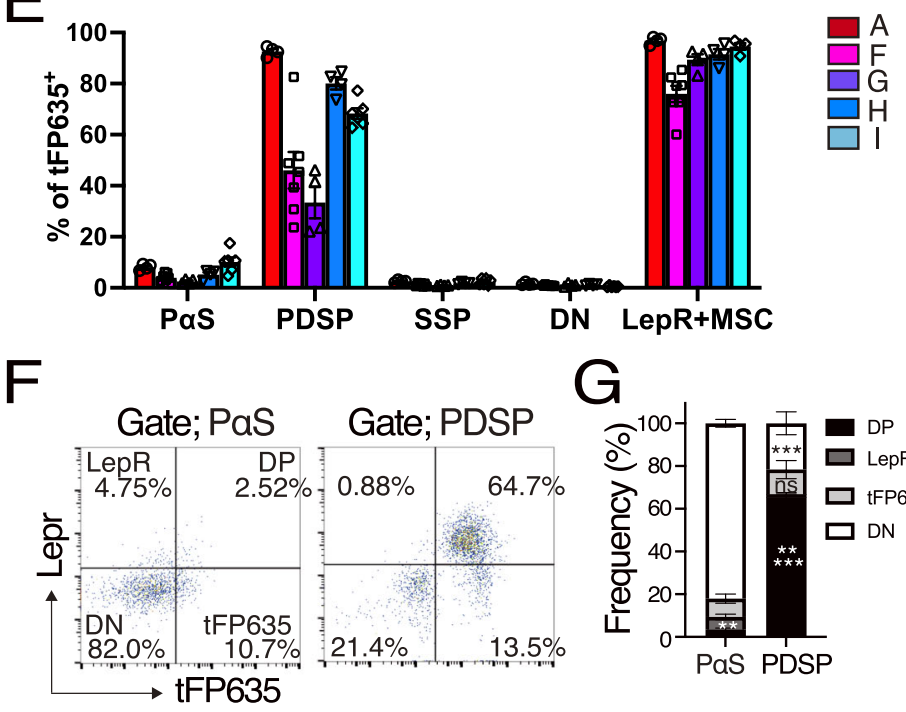

$\mathrm{G}$

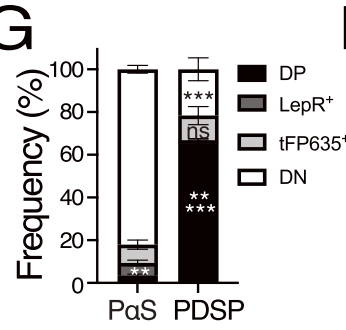

$\mathrm{H}$

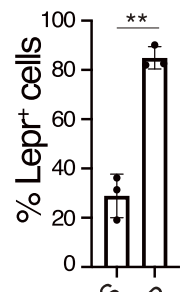

I
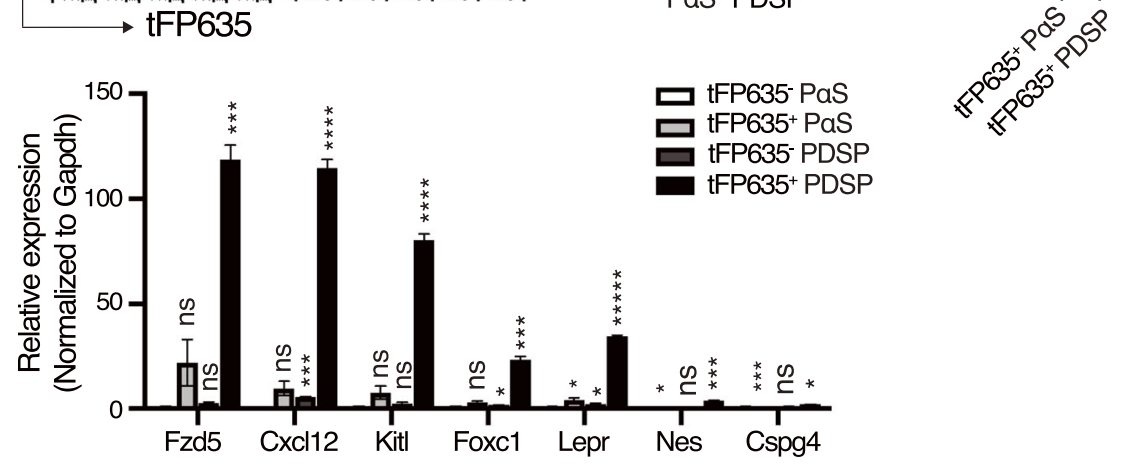

Fig. 2 (See legend on next page.) 


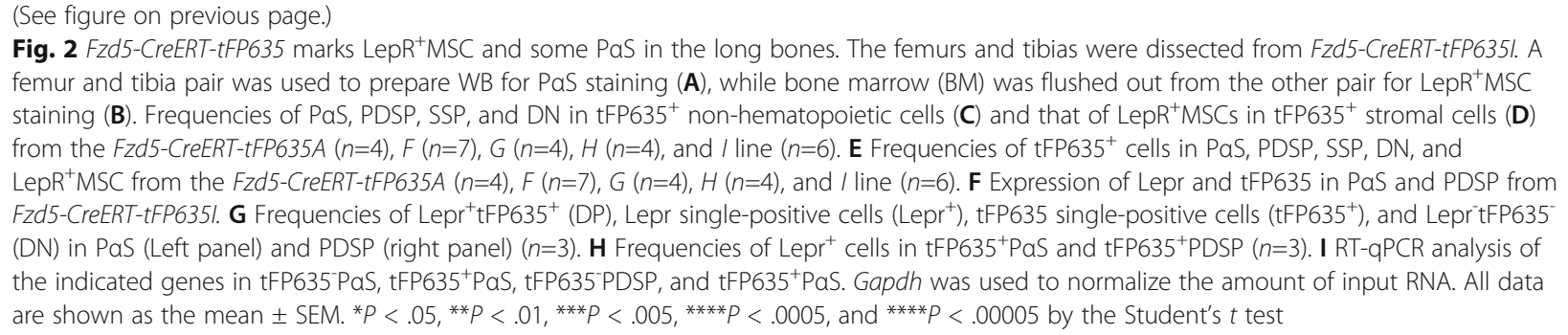

expressed GFP by day 4 , despite over $80 \%$ of LepR ${ }^{+}$MSC was positive for tFP635. GFP positivity slightly increased and was maintained at $60 \%$ for 12 months (Fig. 3C, D). It is known that TM does not necessarily work on all cells, this may explain the discrepancy with the results showing that LepR ${ }^{+}$MSC were largely positive for tFP635 (Fig. 2A-D). We also demonstrated that adipocyte progenitor cells (APC) [20], osteoblast progenitor cells (OPC) [20], and $\mathrm{Cd} 51^{+}$stromal cells were labeled with GFP at 16 weeks after the TM injection in all of the Fzd5-CreERT-tFP635 lines tested (Fig. 3E, F). We note that Fzd5-CreERT-tFP635F, G, and $H$ showed similar results with some variations according to copy number of BAC DNA (Additional file 5). We also analyzed the expression of tFP635 and GFP in the P $\alpha$ S from $F z d 5$-GFP mice 4 weeks after TM injection and showed that more than $70 \%$ of P $\alpha$ S cells were $\mathrm{GFP}^{+}$tFP635 (Fig. 3G).

\section{Lepr-tdTomato also marks PaS subpopulation}

Lep $\mathrm{R}^{+} \mathrm{MSC}$ have been identified as the primary source of bone and adipocytes in adult BM. Lepr-Cre marks LepR ${ }^{+} \mathrm{MSC}$, and lineage tracing showed that LepR ${ }^{+} \mathrm{MSC}$ gave rise to osteoblasts and adipocytes in adult $\mathrm{BM}$ in vivo [7, 8]. Moreover, cell fate mapping with the Ebf3CreERT strain revealed that CAR cells were selfrenewing MSC in adult BM [21]. Similarly, we herein showed that Fzd5-GFP marked LepR ${ }^{+}$MSC with CreERT and tFP635. Therefore, we examined Lepr-Cre; ROSACAG-LSL-tdTomato (hereafter referred to as Lepr-tdTomato) mice at 12 and 24 weeks old to directly compare the identities of the cells labeled in these two different Cre strains (Fig. 4A-C). Consistent with previous findings, virtually all LepR ${ }^{+} \mathrm{MSC}$ were positive for tdTomato in 12-week-old Lepr-tdTomato mice, and the proportion of tdTomato ${ }^{+}$LepR $^{+}$MSC was maintained for another 12 weeks (Fig. 4A, C). In contrast, tdTomato expression was observed in limited $\mathrm{P} \alpha \mathrm{S}$ cells, and the proportion of tdTomato-labeled cells was low even in 24-week-old mice (Fig. 4B, C), while the frequency of $\mathrm{GFP}^{+} \mathrm{P} \alpha \mathrm{S}$ progressively increased in Fzd5-GFP after the TM injection. The data, together with the result that $\mathrm{FP} 635^{+} \mathrm{P} \alpha \mathrm{S}$ cells were largely negative for Lepr, indicates that $\mathrm{tFP} 635^{+} \mathrm{P} \alpha \mathrm{S}$ was distinct subpopulation from tdTomato ${ }^{+} \mathrm{P} \alpha \mathrm{S}$.

\section{Fzd5'PDSP retains MSC properties}

We investigated whether Fzd5-CreERT-tFP635 enable the identification of a novel MSC fraction in BM. We fractionated non-hematopoietic cells from Fzd5-CreERTtFP635I into eight subfractions according to the expression of Sca1, Pdgfra, and tFP635 and tested their colony-forming unit-fibroblast (CFU-F) activities. Among the eight subfractions, tFP $635^{\circ} \mathrm{P} \alpha \mathrm{S}$ exhibited the highest CFU-F activity, followed by tFP635 PDSP and tFP635 ${ }^{+} \mathrm{P} \alpha \mathrm{S}$ in primary plating. tFP635 ${ }^{+} \mathrm{PDSP}$, which consists of LepR ${ }^{+} \mathrm{MSC}$, had no apparent colony-forming capacity. Of note, tFP635 PDSP formed secondary colonies at a similar frequency to the primary colonies, even with marked reductions in the CFU-F activity of tFP635 $\mathrm{P} \alpha \mathrm{S}$ in secondary plating. Moreover, tFP635 PDSP formed colonies even in the third round of plating (Fig. 5A). These data suggest that tFP635-PDSP fraction contains more CFU-Fs with long-term replating capacity than $\mathrm{tFP} 635^{-} \mathrm{P} \alpha \mathrm{S}$ fraction even though its frequency is low. We then examined the differentiation capacity of freshly sorted tFP635 PDSP and found that the cells accumulated lipid droplets less efficient than tFP635 $\mathrm{P} \alpha \mathrm{S}$ in adipogenic induction media (Fig. 5B, C). tFP635 $\mathrm{P} \alpha \mathrm{S}$ accumulated lipid droplets even in the maintenance media, which lacks adipogenic inducers but maintains adipocyte lineage cells, whereas tFP635 PDSP did not (Fig. 5B middle panels). In contrast, osteogenic differentiation was more efficient in tFP635 PDSP. Then, we dissociated the tFP635 $\mathrm{P \alpha S}$ or tFP635 PDSP primary colonies with trypsin and assessed the differentiation capacity. The cells from tFP635'PDSP primary colonies differentiated into adipogenic cells as efficient as freshly sorted tFP635'PDSP, whereas tFP635 $\mathrm{P \alpha S}$ greatly reduced the adipogenic capacity during culture in the colony assay condition (Fig. 5B, C). These data together indicated that cells with a colony-forming capacity in the tFP635 PDSP possess an adipogenic potential in addition to osteogenic potential.

\section{Discussion}

We previously reported that the expression of FZD5 distinguished immature MSC clones with a high proliferative capacity and multilineage differentiation capacity from MSC clones with a limited replicative life span 


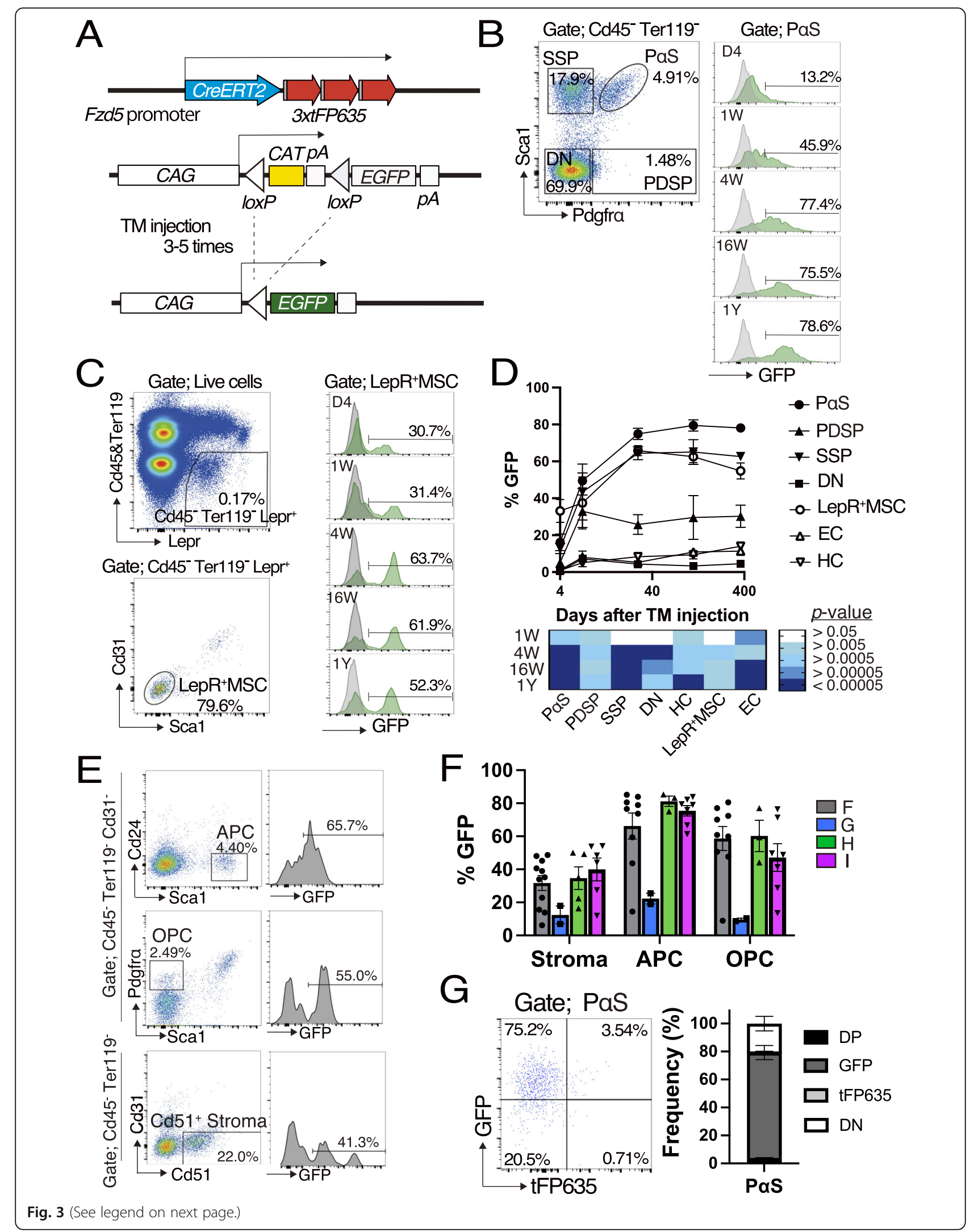


(See figure on previous page.)

Fig. 3 Tracing the lineage of Fzd5-expressing cells. A Strategy for the lineage tracing of Fzd5-expressing cells. B, C Fzd5-GFP were treated with TM at 8-12 weeks of age and the expression of GFP was analyzed in PaS (B) and LepR ${ }^{+} M S C(\mathbf{C})$ at the indicated time points. D Time course changes in GFP positivity in PaS, PDSP, SSP, DN, LepR ${ }^{+} M S C, E C$, and HC are shown $(n=5-10)$. The heat map shows $p$ values against day 4 . $\mathbf{E}$ Frequencies of $\mathrm{GFP}^{+}$cells in APC, OPC, and Cd51 ${ }^{+}$stroma 16 weeks after the TM injection ( $\left.n=9-11\right)$. G Expression of GFP and tFP635 in PaS from Fzd5-GFP 4 weeks after the TM injection ( $n=3)$. All data are shown as the mean \pm SEM. ${ }^{*} P<.05,{ }^{* *} P<.01,{ }^{* * *} P<.005,{ }^{* * *} P<.0005$, and ${ }^{* * * *} P<.00005$ by the Student's $t$ test

in vitro. This finding prompted us to investigate whether the expression of $F z d 5$ also marks immature MSC in vivo, and, thus, we generated the mouse strain, Fzd5CreERT-tFP635, which expresses CreERT and tFP635 in Fzd5-expressing cells. In Fzd5-CreERT-tFP635, the Fzd5 promoter drove tFP635 to LepR ${ }^{+}$MSC and a small fraction of $\mathrm{P} \alpha \mathrm{S}$. LepR ${ }^{+} \mathrm{MSC}$ showed adipogenic, osteogenic, and chondrogenic differentiation in vitro and in vivo and inducible lineage tracing using Ebf3-CreERT also revealed the lifelong self-renewal capacity of LepR ${ }^{+} \mathrm{MSC}$ in the adult stage $[7,8,21]$. Moreover, $\mathrm{P} \alpha \mathrm{S}$ retained trilineage differentiation and self-renewal capacities in vitro. Therefore, $F z d 5$ promoter-driven tFP635 marks two types of previously identified MSC fraction in animal. Recent studies reported that the proliferation and lineage choice of MSC were, at least partially, regulated by systemic and local external signals [22, 23]. Fzd5-

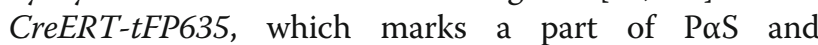
LepR ${ }^{+} M S C$, is a valuable tool for assessing the effects of environmental cues on MSC dynamics. This is the first reporter strain that traces the turnover of $\mathrm{P} \alpha \mathrm{S}$ in a TMinducible manner.

Lepr-tdTomato also marks some $\mathrm{P} \alpha \mathrm{S}$ in addition to LepR ${ }^{+}$MSC. $\quad$ tFP635 ${ }^{+}$PDSP and Lepr-tdTomato ${ }^{+-}$ Lep $\mathrm{R}^{+} \mathrm{MSC}$ appeared to largely overlap because the majority of tFP635 ${ }^{+}$PDSP expressed Lepr; however, we were

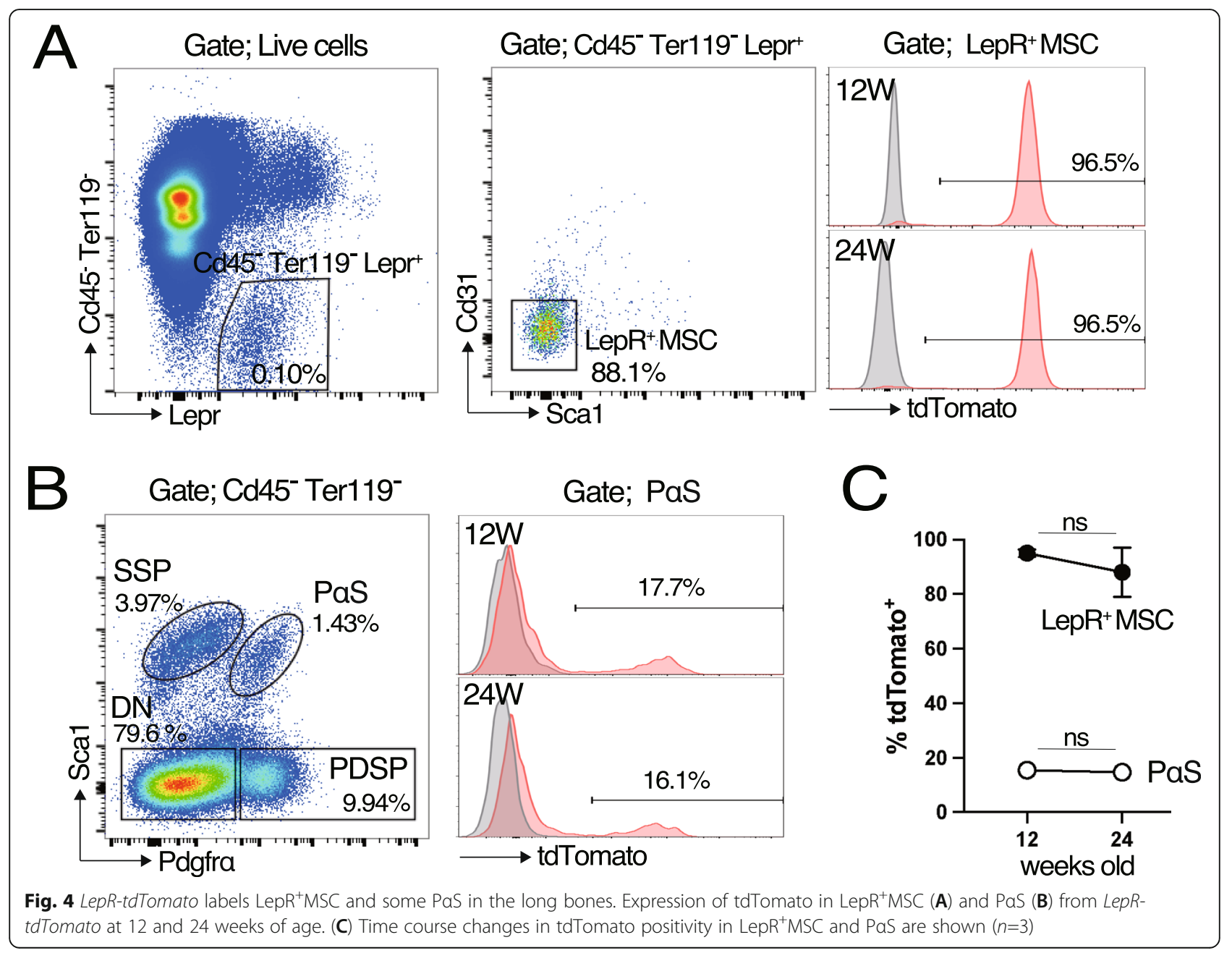




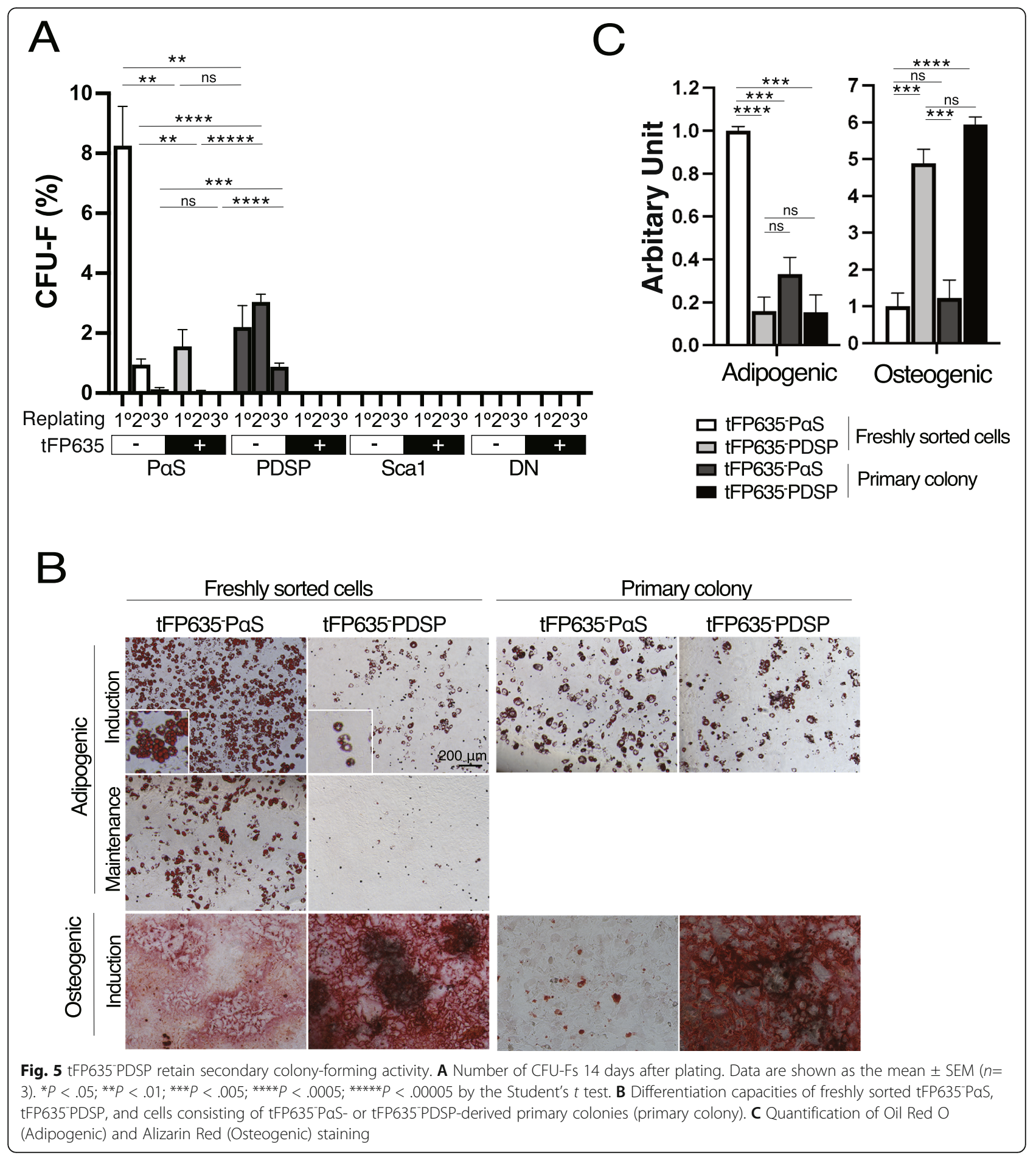

unable to experimentally confirm this due to technical limitations. In contrast, only $25 \%$ of $\mathrm{tFP} 635^{+} \mathrm{P} \alpha \mathrm{S}$ expressed Lepr. In $F z d 5-G F P$, tFP635 ${ }^{+} \mathrm{P} \alpha \mathrm{S}$ accounts for approximately $10 \%$ of $\mathrm{P} \alpha \mathrm{S}$, and the frequency of $\mathrm{GFP}^{+}$ cells progressively increased to approximately $80 \%$ in $\mathrm{P} \alpha \mathrm{S}$ and maintained for a year after the TM injection. In striking contrast, the proportion of tdTomato ${ }^{+}$cells was kept low in P $\alpha S$ during our observation period. Theoretically, at least, a part of $\mathrm{GFP}^{+} \mathrm{P} \alpha \mathrm{S}$ cells were $\mathrm{tFP} 635^{+} \mathrm{P} \alpha \mathrm{S}$ cells or progenies of tFP635 $\mathrm{P} \alpha \mathrm{S}$ cells at early timepoint since GFP labeled cells are CreERT-expressing cells at the time of TM injection or their descendants. Therefore, it seems that Fzd5-CreERT-tFP635 and LeprtdTomato marked distinct $\mathrm{P} \alpha \mathrm{S}$ subpopulations. 
However, we cannot exclude the possibility that some $\mathrm{GFP}^{+} \mathrm{P} \alpha \mathrm{S}$ cells were derived from tFP635 ${ }^{+}$cells outside of the $\mathrm{P} \alpha \mathrm{S}$ fraction in later time-point, e.g., tFP635 PDSP/ LepR ${ }^{+}$MSC or DN, since P $\alpha$ S was not the only cell expressing CreERT in the Fzd5-CreERTtFP635. An approximately $20 \%$ of $P \alpha S$ cells were not labeled with GFP even after 1 year of chasing period in the TM-treated Fzd5-GFP, indicating that these $\mathrm{P} \alpha \mathrm{S}$ cells were quiescent and rarely replaced with $\mathrm{GFP}^{+} \mathrm{P} \alpha \mathrm{S}$ in steady-state or $\mathrm{GFP}^{-} \mathrm{P} \alpha \mathrm{S}$ cells were derived from non$\mathrm{P} \alpha \mathrm{S}$ fraction which do not express the CreERT (discussed below). Development of the mouse strain, which express the Cre recombinase specifically in the P $\alpha$ S, will enable us to understand the hierarchical relationship between the $\mathrm{P} \alpha \mathrm{S}$ and other stromal fractions, e.g., LepR $^{+}$MSC, tFP635 PDSP, or SSP.

We also exhibited that Fzd5-CreERT-tFP635 facilitates screening for non-hematopoietic subfractions in the BM and identified tFP635 PDSP as a putative novel MSC fraction. The colony-forming activity of tFP635 PDSP was approximately $25 \%$ of $\mathrm{tFP} 635^{\circ} \mathrm{P} \alpha \mathrm{S}$ in primary plating, but was sustained or slightly increased in secondary plating and formed a significant amount of tertiary colonies, whereas the CFU-F activities of tFP635 $\mathrm{P} \alpha \mathrm{S}$ and tFP635 ${ }^{+} \mathrm{P} \alpha \mathrm{S}$ markedly decreased in secondary plating. Freshly sorted tFP635 PDSP rarely differentiated into an adipogenic lineage, whereas tFP635 $\mathrm{P} \alpha \mathrm{S}$ robustly accumulated lipid droplets. In contrast, tFP635 PDSP uniformly differentiated into an osteolineage more efficiently than $\mathrm{tFP} 635^{-} \mathrm{P} \alpha \mathrm{S}$. Of note, cells consisting of CFU-F colonies derived from tFP635 PDSP clearly retained adipogenic and osteogenic potentials, showing that only cells with a colony-forming capacity in the tFP635 PDSP fraction possessed an adipogenic potential in addition to osteogenic potential. Therefore, a small portion of tFP635 PDSP is MSC, while the remainders are unipotential cells capable of generating osteolineage cells, thus lineage-biased MSC or osteoblast progenitor. On the other hand, tFP635 $\mathrm{P} \alpha \mathrm{S}$ seems to be a heterogenous population consisting of a small portion of MSC and APC. This result is consistent with previous report showing that $\mathrm{APC}$ and $\mathrm{OPC}$ are immunophenotypically defined as $\mathrm{Cd}^{-} 45^{-} \mathrm{Ter} 19^{-} \mathrm{Cd} 31^{-} \mathrm{Pdgfra}{ }^{+} \mathrm{Cd} 24^{-}$and $\mathrm{Cd}^{-} 5^{-} \mathrm{Ter} 19^{-} \mathrm{Cd} 31^{-} \mathrm{Pdgfra}^{+} \mathrm{Sca} 1^{-}$, respectively [20]. FCM and expression analyses revealed that tFP635 PDSP expressed neither LepR $^{+}$MSC nor Nestin-GFP $P^{+}$MSC markers, demonstrating that tFP635'PDSP were previously unidentified MSC fraction. However, clonogenic in vivo long-term transplantation analyses are needed to confirm this result.

In the E13.5 hindlimb, more than 90\% of nonhematopoietic cells are Pdgfra ${ }^{+} \mathrm{Sca}^{-}$. Some Pdgfra ${ }^{+}$cells start to express Scal as development proceeds, and obvious $\mathrm{P} \alpha \mathrm{S}$ fraction emerged at E18.5 [24]. Moreover, lineage tracing with Pdgfro-CreERT showed that cells exhibiting CFU-F activity in the CR fraction mostly originated from Pdgfra ${ }^{+}$cells [25]. Collectively, these results imply that Pdgfro ${ }^{+} \mathrm{Scal}^{-}$is a precursor of $\mathrm{P} \alpha \mathrm{S}$ in fetal development. Similarly, it is worth to speculate that tFP635 PDSP is a bona fide MSC that give rise to P $\alpha$ S, and also other known MSC, in adults.

\section{Conclusions}

Here, we generated the transgenic mouse strain ( $F z d 5$ CreERT-tFP635) that express the CreERT and tFP635 under the transcriptional control of $F z d 5$ gene using $\mathrm{BAC}$ transgenic technique and showed that the mouse strain marks two different types of MSCs, P $\alpha S$, and LepR $^{+}$MSC.

\section{Abbreviations}

FZD5: Frizzled 5; BAC: Bacterial artificial chromosome; MSC: Mesenchymal stem/stromal cell; BM: Bone marrow; PaS: Pdgfra ${ }^{+} \mathrm{Sca}-1^{+} \mathrm{Cd} 45^{-}$Ter1 $19^{-}$ subfraction; LepR ${ }^{+}$MSC: Leptin receptor-expressing MSCs; CRs: Collagenasereleased cells; FCM: Flow cytometry; PDSP: Pdgfra single-positive cell; SSP: Sca1 single-positive cell; APC: Adipocte prohenitor cell; OPC: Osteoblast progenitor cell; SCF: Stem cell factor; Kit: Kit ligand; CxCl12: C-X-C Motif Chemokine Ligand 12

\section{Supplementary Information}

The online version contains supplementary material available at https://doi. org/10.1186/s41232-022-00194-x.

Additional file 1. Localization of tFP $635^{+}$cells in the femur from Fzd5CreERT-tFP635/ (related to Figure 1). The femur sections were stained with DAPI and observed under a fluorescent microscope. TB, trabecular bone; CB, cortical bone.

Additional file 2. Anatomical localization of $\mathrm{PaS}$ and $\mathrm{LepR}^{+} \mathrm{MSC}$ (related to Figure 2). (A) Schematic representation of the anatomical fractionation experiment. (B) After fractionation of the long bones and enzymatic digestion, a single-cell suspension was stained for PaS (B) or LepR ${ }^{+} M S C$ (C). (D, E) Frequency (D) and absolute number (E) of PaS, PDSP, SSP, and LepR ${ }^{+}$MSC in each fraction. Data are shown as the mean \pm SEM. ${ }^{*} P<.05$; ${ }^{* *} P<.01{ }^{* * *} P<.005 ;{ }^{* * *} P<.0005 ;{ }^{* * * *} P<.00005$ by the Student's $t-$ test.

Additional file 3.. Gating strategy for analysis of Lepr and tFP635 expression in the PaS and PDSP (related to Figure 2). Expression of Lepr and TFP635 in the PaS or PDSP cells from indicated mouse was assessed by FCM

Additional file 4.. Fzd5 expression in the various BM fractions (related to Figure 2). RT-qPCR analysis of Fzd5 in PaS, PDSP, SSP and $\mathrm{Cd} 45^{+}$cells. Hprt1 was used to normalize the amount of input RNA. All data are shown as the mean \pm SEM.

Additional file 5. Lineage analyses of Fzd5-CreERT-tFP635F, G, and $H$ (related to Figure 3). Time course changes in GFP positivity in PaS, PDSP, SSP, DN, LepR ${ }^{+}$MSC, EC, and HC using Fzd5-CreERT-tFP635F ( $\left.n=5-11\right), G$ $(n=3-14)$, and $H(n=4-5)$. Data are shown as the mean \pm SEM.

\section{Acknowledgements}

We would like to thank Hiromi Miyauchi and Mari lizuka for their technical assistance. We are grateful to Dr. Yo Mabuchi (Tokyo Medical and Dental University) for critical reading of the manuscript.

\section{Authors' contributions}

S.M. and Y.K. performed the experiments, analyzed the results, and made the figures; A.W., K.M., R.Y., K.H., and D.H. assisted with the experiments; S.M. and 
Y.M. conceived of and directed the study, secured the funding, and actively wrote the manuscript. All authors read and approved the final manuscript.

\section{Authors' information \\ Satoru Miyagi, Ph.D.}

Position: Associate Professor

E-mail: miyagi@med.shimane-u.ac.jp

Affiliation: Department of Life Science, Faculty of Medicine, Shimane

University

Address: 89-1 Enya, Izumo city, Shimane 693-8501, Japan

Yuko Kato, Ph.D.

Position: Researcher at Affiliation 1 and 2

E-mail: killinblue@gmail.com

Affiliation1: Department of Life Science, Faculty of Medicine, Shimane University,

Address: 89-1 Enya, Izumo city, Shimane 693-8501, Japan

Affiliation2: PuREC Co., Ltd., 89-1 Enya, Izumo city, Shimane 693-8501, Japan.

Address: 89-1 Enya, Izumo city, Shimane 693-8501, Japan

Ayako Watanabe, M.S.

Position: Master's course student

E-mail: am15105@st.kitasato-u.ac.jp

Affiliation: Department of Life Science, Faculty of Medicine, Shimane University

Address: 89-1 Enya, Izumo city, Shimane 693-8501, Japan

Kenichi Miyamoto, Ph.D.

Position: Assistant professor

E-mail: miyaken@med.shimane-u.ac.jp

Affiliation: Department of Life Science, Faculty of Medicine, Shimane

University

Address: 89-1 Enya, Izumo city, Shimane 693-8501, Japan

Rintaro Yoshikawa, Ph.D.

Position: Assistant professor

E-mail: rintaro@med.shimane-u.ac.jp

Affiliation: Department of Life Science, Faculty of Medicine, Shimane

University

Address: 89-1 Enya, Izumo city, Shimane 693-8501, Japan

Keita Hagiya, Ph.D.

Position: Technology Scouting Manager

E-mail: keita.hagiya@fujifilm.com

Affiliation: FUJIFILM Corporation

Address: 9-7-3 Akasaka, Minato-ku, Tokyo 107-0052, Japan

Daisuke Hirano, Ph.D.

Position: Research Manager

E-mail: dskhrn0315@gmail.com

Affiliation: FUJIFILM Corporation

Address: 9-7-3 Akasaka, Minato-ku, Tokyo 107-0052, Japan

Yumi Matsuzaki, M.D. Ph.D.

Position: Professor at Affiliation1 and CSO at Affiliation2

E-mail: matsuzak@med.shimane-u.ac.jp

Affiliation1: Department of Life Science, Faculty of Medicine, Shimane

University,

Address: 89-1 Enya, Izumo city, Shimane 693-8501, Japan

Affiliation2: PuREC Co., Ltd., 89-1 Enya, Izumo city, Shimane 693-8501, Japan.

Address: 89-1 Enya, Izumo city, Shimane 693-8501, Japan

\section{Funding}

This work was supported in part by Grants-in-Aid for Scientific Research (\#19K08865) from MEXT, Japan. This work is also partly supported by the Japanese Society of Hematology Research Grant.

\section{Availability of data and materials}

The data that support the findings of this study and materials are available from the corresponding author, SM, upon reasonable request

\section{Declarations}

\section{Ethics approval and consent to participate}

All experiments using mice were performed under our institutional guidelines for the use of laboratory animals and approved by the Review Board for Animal Experiments of Shimane University (approval ID: IZ30-118 and IZ2-11).

\section{Consent for publication}

Not applicable.

\section{Competing interests}

YK and YM are employee and CSO of PUREC Co., Ltd., respectively. $\mathrm{KH}$ and $\mathrm{DH}$ are employees of FUJIFILM Corporation. The other authors declare that they have no competing interests.

\section{Author details}

'Department of Life Science, Faculty of Medicine, Shimane University, 89-1 Enya, Izumo, Shimane 693-8501, Japan. ${ }^{2}$ PuREC Co., Ltd., Izumo, Shimane, Japan. ${ }^{3}$ FUJIFILM Corporation, Minato-ku, Tokyo, Japan.

Received: 5 October 2021 Accepted: 10 January 2022

Published online: 01 March 2022

\section{References}

1. Pittenger MF, Mackay AM, Beck SC, Jaiswal RK, Douglas R, Mosca JD, et al. Multilineage potential of adult human mesenchymal stem cells. Science. 1999;284(5411):143-7. https://doi.org/10.1126/science.284.5411.143.

2. Li H, Ghazanfari R, Zacharaki D, Lim HC, Scheding S. Isolation and characterization of primary bone marrow mesenchymal stromal cells. Ann NY Acad Sci. 2016:1370(1):109-18. https://doi.org/10.1111/nyas.13102.

3. Mabuchi $Y$, Matsuzaki Y. Prospective isolation of resident adult human mesenchymal stem cell population from multiple organs. Int J Hematol. 2016;103(2):138-44. https://doi.org/10.1007/s12185-015-1921-y.

4. Sugiyama T, Kohara $H$, Noda M, Nagasawa T. Maintenance of the hematopoietic stem cell pool by CXCL12-CXCR4 chemokine signaling in bone marrow stromal cell niches. Immunity. 2006;25(6):977-88. https://doi. org/10.1016/j.immuni.2006.10.016.

5. Omatsu Y, Sugiyama T, Kohara H, Kondoh G, Fujii N, Kohno K, et al. The essential functions of adipo-osteogenic progenitors as the hematopoietic stem and progenitor cell niche. Immunity. 2010;33(3):387-99. https://doi. org/10.1016/j.immuni.2010.08.017

6. Yue R, Zhou BO, Shimada IS, Zhao Z, Morrison SJ. Leptin receptor promotes adipogenesis and reduces osteogenesis by regulating mesenchymal stromal cells in adult bone marrow. Cell Stem Cell. 2016;18(6):782-96. https://doi. org/10.1016/j.stem.2016.02.015.

7. Zhou BO, Yue R, Murphy MM, Peyer JG, Morrison SJ. Leptin-receptorexpressing mesenchymal stromal cells represent the main source of bone formed by adult bone marrow. Cell Stem Cell. 2014;15(2):154-68. https://doi. org/10.1016/j.stem.2014.06.008

8. Zhou BO, Yu H, Yue R, Zhao Z, Rios JJ, Naveiras O, et al. Bone marrow adipocytes promote the regeneration of stem cells and haematopoiesis by secreting SCF. Nat Cell Biol. 2017. https://doi.org/10.1038/ncb3570.

9. Ding L, Morrison SJ. Haematopoietic stem cells and early lymphoid progenitors occupy distinct bone marrow niches. Nature. 2013:495(7440): 231-5. https://doi.org/10.1038/nature11885.

10. Ding L, Saunders TL, Enikolopov G, Morrison SJ. Endothelial and perivascular cells maintain haematopoietic stem cells. Nature. 2012;481(7382):457-62. https://doi.org/10.1038/nature10783.

11. Pinho S, Lacombe J, Hanoun M, Mizoguchi T, Bruns I, Kunisaki Y, et al. PDGF Ra and CD51 mark human Nestin+ sphere-forming mesenchymal stem cells capable of hematopoietic progenitor cell expansion. J Exp Med. 2013:210(7): 1351-67. https://doi.org/10.1084/jem.20122252.

12. Dominici M, Le Blanc K, Mueller I, Slaper-Cortenbach I, Marini F, Krause D, et al. Minimal criteria for defining multipotent mesenchymal stromal cells. The International Society for Cellular Therapy position statement. Cytotherapy. 2006. https://doi.org/10.1080/14653240600855905.

13. Uccelli A, Moretta L, Pistoia V. Mesenchymal stem cells in health and disease. Nat Rev Immunol. 2008;8(9):726-36. https://doi.org/10.1038/nri2395.

14. Morikawa S, Mabuchi $Y$, Kubota $Y$, Nagai $Y$, Niibe $K$, Hiratsu E, et al. Prospective identification, isolation, and systemic transplantation of multipotent mesenchymal stem cells in murine bone marrow. J Exp Med. 2009:206(11):2483-96. https://doi.org/10.1084/jem.20091046.

15. Harada S, Mabuchi Y, Kohyama J, Shimojo D, Suzuki S, Kawamura Y, et al. FZD5 regulates cellular senescence in human mesenchymal stem/stromal cells. Stem Cells. 2021;39(3):318-30. https://doi.org/10.1002/stem.3317.

16. Kawamoto S, Niwa H, Tashiro F, Sano S, Kondoh G, Takeda J, et al. A novel reporter mouse strain that expresses enhanced green fluorescent protein 
upon Cre-mediated recombination. FEBS Lett. 2000;470(3):263-8. https://doi. org/10.1016/s0014-5793(00)01338-7.

17. Kawamoto $\mathrm{T}$. Use of a new adhesive film for the preparation of multipurpose fresh-frozen sections from hard tissues, whole-animals, insects and plants. Arch Histol Cytol. 2003;66(2):123-43. https://doi.org/10.1679/aohc. 66.123.

18. Crane G, Jeffery E, Morrison SJ. Adult haematopoietic stem cell niches. Nat Rev Immunol. 2017. https://doi.org/10.1038/nri.2017.53.

19. Omatsu Y, Seike M, Sugiyama T, Kume T, Nagasawa T. Foxc1 is a critical regulator of haematopoietic stem/progenitor cell niche formation. Nature. 2014;508(7497):536-40. https://doi.org/10.1038/nature13071.

20. Ambrosi TM, Scialdone A, Graja A, Gohlke S, Jank AM, Bocian C, et al. Adipocyte accumulation in the bone marrow during obesity and aging impairs stem cell-based hematopoietic and bone regeneration. Cell Stem Cell. 2017:20(6):771-784.e6. https://doi.org/10.1016/j.stem.2017.02.009.

21. Seike $M$, Omatsu $Y$, Watanabe $H$, Kondoh $G$, Nagasawa T. Stem cell nichespecific Ebf3 maintains the bone marrow cavity. Genes Dev. 2018;32(5-6): 359-72. https://doi.org/10.1101/gad.311068.117.

22. Thomas $\mathrm{S}$, Jaganathan BG. Signaling network regulating osteogenesis in mesenchymal stem cells. J Cell Commun Signal. 2021;16(1):47-61. https:// doi.org/10.1007/s12079-021-00635-1.

23. Hawkes CP, Mostoufi-Moab S. Fat-bone interaction within the bone marrow milieu: Impact on hematopoiesis and systemic energy metabolism. Bone. 2019;119:57-64. https://doi.org/10.1016/j.bone.2018.03.012.

24. Nusspaumer G, Jaiswal S, Barbero A, Reinhardt R, Ronen DI, Haumer A, et al. Ontogenic identification and analysis of mesenchymal stromal cell populations during mouse limb and long bone development. Stem Cell Rep. 2017;9(4):1124-38. https://doi.org/10.1016/j.stemcr.2017.08.007.

25. Miwa H, Era T. Tracing the destiny of mesenchymal stem cells from embryo to adult bone marrow and white adipose tissue via Pdgfra expression. Development. 2018. https://doi.org/10.1242/dev.155879 PMID: 29378823.

\section{Publisher's Note}

Springer Nature remains neutral with regard to jurisdictional claims in published maps and institutional affiliations.

Ready to submit your research? Choose BMC and benefit from:

- fast, convenient online submission

- thorough peer review by experienced researchers in your field

- rapid publication on acceptance

- support for research data, including large and complex data types

- gold Open Access which fosters wider collaboration and increased citations

- maximum visibility for your research: over $100 \mathrm{M}$ website views per year

At $\mathrm{BMC}$, research is always in progress.

Learn more biomedcentral.com/submissions 